\title{
MAGNETARS AS HIGHLY MAGNETIZED QUARK STARS: AN ANALYTICAL TREATMENT
}

\author{
M. Orsaria ${ }^{1,2}$, Ignacio F. Ranea-SAndoval ${ }^{1,2}$, And H. Vucetich ${ }^{1}$ \\ ${ }^{1}$ Gravitation, Astrophysics and Cosmology Group, Facultad de Ciencias Astronómicas y Geofísicas, Universidad Nacional de La Plata UNLP, \\ Paseo del Bosque S/N (1900), La Plata, Argentina \\ ${ }^{2}$ CONICET, Rivadavia 1917, 1033 Buenos Aires, Argentina \\ Received 2010 October 15; accepted 2011 March 30; published 2011 May 24
}

\begin{abstract}
We present an analytical model of a magnetar as a high-density magnetized quark bag. The effect of strong magnetic fields $\left(B>5 \times 10^{16} \mathrm{G}\right)$ in the equation of state is considered. An analytic expression for the mass-radius relationship is found from the energy variational principle in general relativity. Our results are compared with observational evidence of possible quark and/or hybrid stars.
\end{abstract}

Key words: dense matter - magnetic fields - methods: analytical - stars: individual (Quark Stars) - stars: magnetars

\section{INTRODUCTION}

The fundamental aspects of the physics involved in the description of the matter inside a white dwarf are well understood (Shapiro \& Teukolsky 1983), but in the case of neutron stars the situation is rather different because the equation of state (EoS) of neutron matter at very high densities is still unknown.

The interior of a neutron star is an astrophysical laboratory in which matter is compressed to high densities. The compression of matter several times the saturation nuclear matter density, $\rho_{0}$, may produce a phase transition from nuclear to quark matter, i.e., an unconfined quark-gluon plasma. In addition, under suitable circumstances, a conversion $\mathrm{d} \rightarrow \mathrm{s}$ quarks may happen through weak interactions, leading to what has been called strange quark matter (SQM). It has been stated that SQM may be the absolute ground state of strong interactions (Bodmer 1971; Witten 1984), although such a hypothesis has not been confirmed yet. The natural scenario where SQM could occur is the inner core of neutron stars. Hence, if the SQM hypothesis is true, some neutron stars could be either hybrid stars, which have quark cores surrounded by a hadronic shell, or quark stars. Already 40 years ago, the existence of quark stars in hydrostatic equilibrium was suggested by Itoh (1970) in a preliminary work.

On the other hand, it is well known that at the surface of neutron stars there exist magnetic fields of the order of $10^{12}-10^{13} \mathrm{G}$. Compact stars with ultra strong magnetic fields $\left(10^{2}-10^{3}\right.$ larger than those of a typical neutron star) are called magnetars. In such objects the magnetic field at the surface could be higher than $10^{15} \mathrm{G}$ (de la Incera 2009).

Knowledge of the magnetars' composition would help explain some astrophysical phenomena. Soft gamma-ray repeaters (SGRs) and anomalous X-ray pulsars (AXPs) have been interpreted as evidence of magnetars. Besides, some authors (Cheng \& Daib 2002; Ouyed et al. 2007) claim that magnetized hybrid or quark stars could be the real sources of SGRs and AXPs. The mass-radius $(M-R)$ relationship tells us how matter composing the star behaves under compression, providing information about its composition. Several EoS for neutron, hybrid, and quark stars have been proposed but none of them is conclusive (Douchin \& Haensel 2001; Lattimer \& Prakash 2001, 2007; Özel \& Psaltis 2009). Each EoS produces a different $M-R$ relationship which can be contrasted with the available observational data in order to test its range of validity and/or set bounds on some parameters. At this particular point astrophysical studies become of great importance since they could shed some light on understanding fundamental aspects of matter: microphysics could be inferred from macrophysics. Here lies the great importance of the studies related to ultracompact objects. For instance, Lattimer \& Prakash $(2001,2007)$ contrast some $M-R$ relationships obtained theoretically for different EoS. By varying some parameters a difference of $4 \%-10 \%$ and $10 \%-15 \%$ in determining the maximum radius $R_{\max }$ and mass $M_{\max }$, respectively, is shown for the same EoS.

Several papers (Chakrabarty \& Sahu 1996; González Felipe \& Pérez Martínez 2009; Pérez Martínez et al. 2010) study the $M-R$ relationship of highly magnetized quark stars (HMQS) through numerical integration of the Tolman-Oppenheimer-Volkoff equation for different EoS. Although most studies of quark stars' properties have used such methods, Banerjee et al. (2000) have obtained a maximum mass and radius for unmagnetized quark stars analytically by using a non-relativistic gravitational treatment.

Approximate analytical solutions play an important role in the astrophysical analysis, giving a keener insight than the numerical solutions. Moreover, they may be used as a testing point to check if the numerical scheme is accurate and also they are the first step in the comparison between theory and observation. Indeed, an approximate analytical solution for the $M-R$ relationship may be all that is required when comparisons with observational limits that determine the confidence contour for the mass and radius are performed. Besides, in the highdensity EoS the uncertainties are of the same order as or larger than the errors in the variational method.

The appropriate treatment for quark stars should be relativistic, since the existence of a maximum mass is associated with the behavior of a relativistic gas and general relativistic corrections are dominant (Weinberg 1972). In this paper, we shall use the general relativistic energy variational principle described by Naurenberg \& Chapline (1973) to obtain an analytic approximate formula for the mass, radius, and baryonic number of an HMQS. Quark stars are particularly suitable for a variational treatment since their density profile resembles a constant mass density star. We shall model an HMQS assuming quark matter within a high density regime in the framework of a modified MIT Bag model EoS. We also assume that the magnetic field $B$ is low enough to be treated like a correction in 
the $\operatorname{EoS}$ ( $B \ll \mu^{2}$, with $\mu$ being the baryon chemical potential) although, as we will see in the following sections, this is not a strong restriction.

The paper is organized as follows. In Section 2, we calculate the thermodynamical quantities of the system and analyze the stability of quark matter with respect to decomposition in baryons. In Section 3, we provide the analytic relativistic $M-R$ relationship and compare our results with the observational data. We also check the dynamic stability of the star by calculating the adiabatic index and the speed of sound. In Section 4, we present a summary of our main results and conclusions.

\section{HIGH-DENSITY QUARK MATTER WITHIN A STRONG MAGNETIC FIELD}

In this section we shall discuss the analytic approximations to the SQM EoS in the presence of a uniform magnetic field $\boldsymbol{B} \| \hat{z}$. Within the framework of the MIT Bag model, we assume three massless quarks $\mathrm{u}, \mathrm{d}$, and $\mathrm{s}$, neglecting mediated interactions between them. We also consider that the strong magnetic field is a small contribution to the total energy, a fact that will be checked later.

\subsection{Quark Matter in a Magnetic Field}

Let us compute the grand canonical thermodynamic potential $\Omega$ in the high density regime. Due the Landau quantization the phase space volume integral in the momentum space is replaced by

$$
\begin{aligned}
\frac{1}{(2 \pi)^{3}} \int d^{3} p f(p) & =\frac{1}{(2 \pi)^{3}} \int d p_{z} d^{2} p_{\perp} f(p) \\
& =\frac{q B}{4 \pi^{2}} \sum_{\nu=0}^{\nu=\infty}\left(2-\delta_{\nu 0}\right) \int_{-\infty}^{+\infty} d p_{z} f\left(\nu, p_{z}\right)
\end{aligned}
$$

where $\left(2-\delta_{\nu 0}\right)$ means that the zeroth Landau level is singly degenerate, whereas all other states are doubly degenerate. The grand canonical potential for each quark in the presence of a strong magnetic field is given by

$$
\begin{aligned}
\Omega_{i}= & -\frac{q_{i} B g_{i}}{8 \pi^{2}} \sum_{\nu=0}^{\nu_{\max }}\left(2-\delta_{\nu 0}\right)\left[\mu \sqrt{\mu^{2}-2 \nu q_{i} B}\right. \\
& \left.-2 \nu q_{i} B \ln \frac{\mu+\sqrt{\mu^{2}-2 v q_{i} B}}{\sqrt{2 \nu q_{i} B}}\right],
\end{aligned}
$$

where $g_{i}=2 \times 3$, taking into account spin and color degeneracy, and $q_{i}$ is the absolute value of the charge of the particle, $q_{u}=2|e| / 3$ and $q_{d}=q_{s}=|e| / 3$, with $e$ being the value of electronic charge.

For simplicity, we consider the quark masses $m_{q}=0$, which implies that the electrons are not present and the quarks' chemical potentials are, as a consequence of equilibrium conditions, all equal, $\mu_{u}=\mu_{d}=\mu_{s} \equiv \mu$.

By imposing that

$$
p_{z}^{2}=\mu^{2}-2 v q_{i} B \geqslant 0,
$$

we can determine the upper limit of the sum $v_{\max }$ from

$$
v \leqslant \frac{\mu^{2}}{2 q_{i} B} \equiv v_{\max } .
$$

The series, Equation (2), can be approximated using the Euler-MacLaurin formula

$$
\begin{aligned}
\sum_{j=0}^{n} f(j)= & \int_{0}^{n} f(x) d x+\frac{1}{2}[f(n)+f(0)] \\
& +\frac{1}{12}\left[f^{\prime}(n)-f^{\prime}(0)\right]-\frac{1}{720}\left[f^{\prime \prime \prime}(n)-f^{\prime \prime \prime}(0)\right]+R,
\end{aligned}
$$

where the remainder term, $R$, usually is expressed in terms of periodic Bernoulli polynomials (Spivey 2006) and can be estimated by using

$$
|R| \leqslant \frac{2 \zeta(4)}{(2 \pi)^{4}} \int_{1}^{v_{\max }-1}\left|f^{\mathrm{IV}}(\nu)\right| d \nu,
$$

where the Riemann Zeta function $\zeta(4) \simeq 1.0823$. To avoid divergences appearing in the third term of Equation (5), in the limit of high densities or negligible quark masses, we apply the Euler-MacLaurin formula in the form

$$
\begin{aligned}
\Omega_{i} \simeq & \Omega_{i}\left(v_{\max }\right)+\Omega_{i}(0)+\int_{1}^{v_{\max }-1} \Omega_{i}(v) d v \\
& +\frac{1}{2}\left[\Omega_{i}\left(v_{\max }-1\right)+\Omega_{i}(1)\right] \\
& +\frac{1}{12}\left[\left.\frac{\partial \Omega_{i}}{\partial v}\right|_{\left(v_{\max }-1\right)}-\left.\frac{\partial \Omega_{i}}{\partial v}\right|_{(1)}\right]+\tilde{R},
\end{aligned}
$$

where

$$
\tilde{R}=-\frac{1}{720}\left[\left.\frac{\partial^{3} \Omega_{i}}{\partial \nu^{3}}\right|_{\left(v_{\max }-1\right)}-\left.\frac{\partial^{3} \Omega_{i}}{\partial \nu^{3}}\right|_{(1)}\right]+R .
$$

We have considered Equation (8) as the remainder term, which gives $|\tilde{R}| \leqslant 3 \%$. In the limit $\mu^{2} \gg 2 q_{i} B$, the thermodynamical potential can be calculated by performing first the integral in Equation (7) and then expanding in power series of $B$. The result is

$$
\Omega_{i}=-\frac{\mu^{4}}{4 \pi^{2}}+\frac{q_{i}^{2} B^{2}}{8 \pi^{2}}\left(\log \frac{q_{i} B}{2 \mu^{2}}-3\right)+\mathcal{O}\left(B^{5 / 2}\right) .
$$

The particle density $n_{i}=-\frac{\partial \Omega_{i}}{\partial \mu}$ is

$$
n_{i}=\frac{\mu^{3}}{\pi^{2}}+\frac{q_{i}^{2} B^{2}}{4 \pi^{2} \mu}+\mathcal{O}\left(B^{5 / 2}\right) .
$$

Note that when $B=0$ in Equations (9) and (10), we recover the usual expressions for a non-interacting massless quark gas at zero temperature and zero magnetic field.

\subsection{Equation of State}

With the above results, one can form the modified EoS of SQM in the MIT Bag model. Within this framework, the difference between the energy density of the perturbative and non-perturbative QCD vacuum is taken into account by the "bag constant" $B_{\text {bag }}$. Considering $\hbar=c=1$, we can find the conversion factor between high energy density units and magnetic energy density units. We can write $1 \mathrm{MeV} \simeq$ $1.6 \times 10^{-6}$ erg and $1 \mathrm{MeV} \simeq\left(2 \times 10^{-11} \mathrm{~cm}\right)^{-1}$, where 
$1 \mathrm{MeV}^{4} \simeq 2 \times 10^{26} \mathrm{erg} \mathrm{cm}^{-3}$. Relating this quantity with the magnetic energy density $B^{2} / 8 \pi$, the conversion factor for the magnetic field is given by $1.4 \times 10^{13} \mathrm{G} \equiv 1 \mathrm{MeV}^{2}$.

The charge neutrality condition

$$
2 n_{u}=n_{d}+n_{s}
$$

and the $\beta$-equilibrium condition

$$
\mu_{u}=\mu_{d}=\mu_{s} \equiv \mu
$$

are automatically satisfied.

Combining the results of Section 2, we obtain

$$
\begin{aligned}
\Omega= & \sum_{i=u, d, s} \Omega_{i}+B_{\mathrm{eff}}=-\frac{3 \mu^{4}}{4 \pi^{2}}+\frac{B^{2}}{12 \pi^{2}} \\
& \times\left(\log \frac{B}{2^{1 / 3} 3 \mu^{2}}-3\right)+B_{\mathrm{eff}}+\mathcal{O}\left(B^{5 / 2}\right),
\end{aligned}
$$

where $B_{\text {eff }}=B^{2} / 8 \pi+B_{\text {bag }}$. Replacing Equation (11) in the baryon number density condition, $n_{B}=\frac{1}{3} \sum_{i=u, d, s} n_{i}$, we obtain

$$
n_{B}=\frac{\mu^{3}}{\pi^{2}}+\frac{B^{2}}{9 \pi^{2} \mu}+\mathcal{O}\left(B^{5 / 2}\right) .
$$

Since we work in the $T=0$ limit, the energy density is given by

$$
\begin{aligned}
\rho=\Omega+3 \mu n_{B}= & \frac{9 \mu^{4}}{4 \pi^{2}}+\frac{B^{2}}{12 \pi^{2}}\left(1+\log \frac{B}{2^{1 / 3} 3 \mu^{2}}\right) \\
& +B_{\text {eff }}+\mathcal{O}\left(B^{5 / 2}\right),
\end{aligned}
$$

whereas the pressure reads

$P=-\Omega=\frac{3 \mu^{4}}{4 \pi^{2}}-\frac{B^{2}}{12 \pi^{2}}\left(\log \frac{B}{2^{1 / 3} 3 \mu^{2}}-3\right)-B_{\text {eff }}+\mathcal{O}\left(B^{5 / 2}\right)$.

Note that we are not considering the anisotropy of pressures (González Felipe et al. 2008) because we are working in the limit of weak magnetic field, $\mu^{2} \gg 2 q_{i} B$. The relation between the total energy density, Equation (15), and the total pressure, Equation (16), determines the EoS of the system as

$$
\rho=3 P+4 B_{\text {eff }}-\frac{B^{2}}{3 \pi^{2}}\left(2-\log \frac{B}{2^{1 / 3} 3 \mu^{2}}\right)+\mathcal{O}\left(B^{5 / 2}\right) .
$$

\subsection{Stability Analysis: Strong Interactions}

It is well known that SQM may be stable with respect to decay into nucleons at zero pressure and zero temperature if its energy per baryon $\rho / n_{B}$ is less than the energy per baryon of ${ }^{56} \mathrm{Fe}=930 \mathrm{MeV}$ (Farhi \& Jaffe 1984). The presence of a magnetic field changes this stability condition somewhat.

At $P=0$ we can estimate the chemical potential through the successive approximation method as

$$
\mu\left(B, B_{\text {bag }}\right)=\left[\frac{4 \pi^{2} B_{\text {eff }}}{3}+\frac{B^{2}}{3^{2}}\left(\log \frac{B}{2^{4 / 3} \pi \sqrt{3 B_{\text {bag }}}}-3\right)\right]^{1 / 4},
$$

which will be replaced in Equations (14) and (17) to evaluate $\frac{\rho}{n_{B}}$. Contrary to previous results (Anand \& Singh 1999; Chakrabarty 1999; González Felipe \& Pérez Martínez 2009), we find that
Table 1

Bag Constant, Baryon Density, and Magnetic Field Upper Limit to Preserve Quark Matter Stability Condition

\begin{tabular}{lcc}
\hline \hline$B_{\text {bag }}\left(\mathrm{MeV} \mathrm{fm}^{-3}\right)$ & $n_{B} / n_{0}$ & $B_{\max }(\mathrm{G})$ \\
\hline 57 & $1.73 \pm 0.01$ & $4.4 \times 10^{17}$ \\
60 & $1.80 \pm 0.01$ & $4.8 \times 10^{17}$ \\
75 & $2.14 \pm 0.01$ & $5.3 \times 10^{17}$ \\
80 & $2.24 \pm 0.02$ & $5.5 \times 10^{17}$ \\
85 & $2.34 \pm 0.02$ & $5.7 \times 10^{17}$ \\
90 & $2.45 \pm 0.02$ & $5.8 \times 10^{17}$ \\
\hline
\end{tabular}

the energy per baryon increases with $B$ (Equation (17)). The condition $\rho / n_{B}<930 \mathrm{MeV}$ is satisfied for magnetic fields $B<4.4 \times 10^{18} \mathrm{G}$. However, for the stability of the system, not only it is necessary to consider the energy per baryon, but also the influence of magnetic energy density. We obtain $B^{2} / 8 \pi \sim B_{\text {bag }}$ for $85 \mathrm{MeV} \mathrm{fm}^{-3}<B_{\mathrm{bag}}<90 \mathrm{MeV} \mathrm{fm}^{-3}$ and $B^{2} / 8 \pi>B_{\text {bag }}$ for $57 \mathrm{MeV} \mathrm{fm}^{-3}<B_{\text {bag }}<80 \mathrm{MeV} \mathrm{fm}^{-3}$. Although it is known that the binding of the quark stars is provided not by gravitation, but rather by the strong interactions, the inclusion of a magnetic field $B$ adds an additional constraint to the stability condition through the magnetic energy density and the magnetic pressure. When the latter is not lower than $B_{\mathrm{bag}}$, the magnetic field becomes dynamically important. Furthermore if the magnetic pressure is of the same order of magnitude as the matter pressure, spherical deformation effects should be considered. In addition, suficiently strong magnetic fields can generate an anisotropic pressure distribution inside the HMQS modifying the EoS and consequently the $M-R$ relationship (Paulucci et al. 2011). Therefore, for magnetic fields large enough, $B_{\text {eff }}$ is greater than the kinetic energy of the quarks thereby destabilizing the star. Thus, we also consider the relationship

$$
\left(B^{2} / 8 \pi\right) B_{\text {bag }}^{-1}<0.1
$$

to guarantee both the perturbative treatment of the system and the stability of the star. Table 1 shows that the variation of baryon density is quite small for $P=0$ when increasing the magnetic field from 0 up to $B_{\max }$.

\section{MASS-RADIUS RELATIONSHIP BY VARIATIONAL METHOD}

The energy variational method in general relativity is explained in detail in Harrison et al. (1965; see also Weinberg 1972). Starting from a uniform density configuration in a spherically symmetric distribution the total mass $M$, the baryon number $N_{B}$ and the radius $R$ of the star are given by

$$
\begin{aligned}
M & =\frac{4}{3} \pi \rho R^{3}, \\
N_{B} & =2 \pi n_{B} a^{3}(\chi-\sin \chi \cos \chi), \\
R & =a \sin \chi,
\end{aligned}
$$

where $\rho$ is the mass-energy density and the angle $\chi$ comes from substituting $r=a \sin \chi$, where $a=[3 /(8 \pi \rho)]^{1 / 2}$ is the curvature radius in the metric inside the star which adopts the following form for the 3-geometry:

$$
d s^{2}=a^{2}\left[d \chi^{2}+\sin ^{2} \chi\left(d \theta^{2}+\sin ^{2} \theta d \phi^{2}\right)\right] .
$$

Note that we are using $\hbar=c=G=1$. The configuration of maximum density is achieved when $\chi=\pi / 2$. Observe that 
$\sin ^{2} \chi=2 M / R, \chi \sim 0$ corresponds to the Newtonian limit, while $\chi=\pi / 2$ corresponds to the Schwarzschild one. The use of a constant energy trial configuration has been justified by Naurenberg \& Chapline (1973), while Weinberg (1972) has applied it to white dwarfs. In this latter case, a fair approximation to the Chandrasekhar mass was obtained.

To obtain the equilibrium condition it is appropriate to treat $\chi$ as an independent variable. Imposing $\partial M / \partial \chi=0$ for fixed $N_{B}$, the equilibrium condition reads

$$
w \equiv \frac{P}{\rho}=\zeta(\chi)
$$

where $\rho$ and $P$ are given by Equations (15) and (16), and $\zeta(\chi)$ is a function independent of the EoS:

$$
\zeta(\chi)=3 \cos \chi\left(\frac{9}{2} \cos \chi-\frac{\sin ^{3} \chi}{\chi-\sin \chi \cos \chi}\right)^{-1}-1 .
$$

We get an approximate value of $\zeta(\chi)$ using a Taylor series, $\zeta_{T}$, around $\chi=0$. Truncation at eighth order gives

$$
\zeta_{T}=\frac{1}{10} \chi^{2}+\frac{113}{2100} \chi^{4}+\frac{1747}{63,000} \chi^{6}+\frac{689,687}{48,510,000} \chi^{8}
$$

The Padé approximant of order $(4,4)$ gives a representation of this function that is also an approximate analytic continuation beyond the circle of convergence. Thus, $\zeta(\chi)$ is given as a ratio of two polynomials as

$\zeta_{P}=\left(-\frac{23}{6237} \chi^{4}+\frac{1}{10} \chi^{2}\right)\left(1-\frac{5123}{8910} \chi^{2}+\frac{3002}{93,555} \chi^{4}\right)^{-1}$.

Imposing $\zeta_{P}=w$ we obtain the only physical solution for $\chi$, always positive and fulfilling the condition $\lim _{w \rightarrow 0} \chi=0$, given by

$$
\begin{aligned}
& \chi=\frac{\sqrt{3}}{2} \\
& \times \frac{\sqrt{\left(35861 w+6237-\sqrt{\left.786718681 w^{2}+389949714 w+38900169\right)}\right.}}{\sqrt{3002 w+345}} .
\end{aligned}
$$

Hence, we get an analytical expression of the HMQS mass and radius as a function of the baryonic chemical potential. This allows us to obtain the $M-R$ relationship for different bag constant and magnetic field values. In particular, in Figure 1 we show the $B_{\text {bag }}=57 \mathrm{MeV} \mathrm{fm}^{-3}$ and $B_{\text {bag }}=90 \mathrm{MeV} \mathrm{fm}^{-3}$ cases with and without magnetic field. Note that for the first one, when $B=0$, the $M-R$ curve coincides with the hadronic star zone. This result can be attributed to the fact that the value $B_{\text {bag }}=57 \mathrm{MeV} \mathrm{fm}^{-3}$ could be too low for a quark star which has been modeled by using the MIT bag model (Zdunik et al. 2000). Furthermore, in Table 2 we present the results for other values of $B_{\mathrm{bag}}$. Note that although $B_{\max }$ is a typical value for a magnetar it slightly decreases the $M_{\max }$ if compared with the zero magnetic field case. The same result is obtained for $R_{\max }$.

\subsection{Dynamical Stability}

In our model the condition for stable equilibrium is given by $\partial^{2} M / \partial^{2} \chi>0$. For a given EoS, it is possible to determine

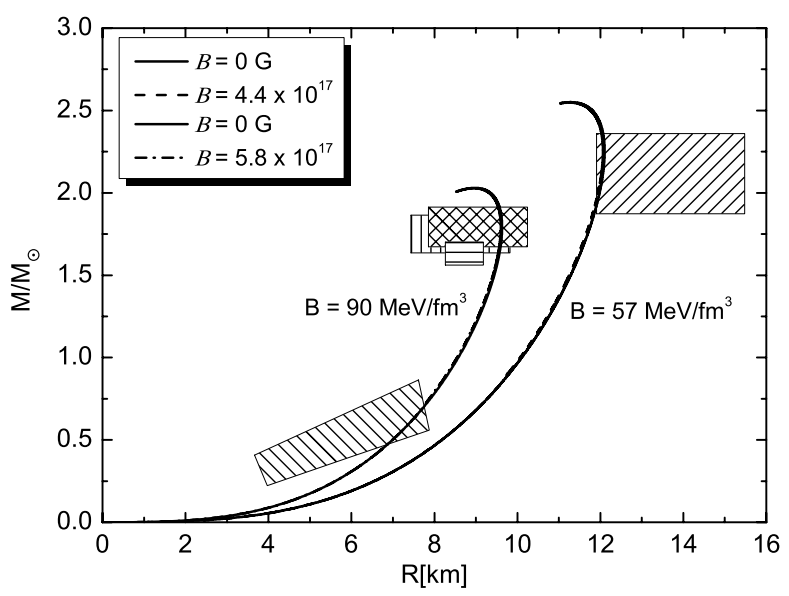

Figure 1. Mass-radius relationship with and without magnetic field for $B_{\mathrm{bag}}=57 \mathrm{MeV} \mathrm{fm}^{-3}$ (solid and dashed lines) and $B_{\mathrm{bag}}=90 \mathrm{MeV} \mathrm{fm}^{-3}$ (solid and dash-dotted line). The rectangle with diagonal pattern corresponds to EXO 0748-676, which has been interpreted as a hadronic star. Rectangles with crossed, vertical, and horizontal patterns correspond to quarks or hybrid stars (Drago \& Lavagno 2010). The polygon could be a low-mass strange star as suggested in Zhang et al. (2007).

the quark densities and pressures where quark stars are stable against gravitational collapse from the condition

$$
\Gamma>\Gamma_{c}
$$

where the adiabatic index for SQM, $\Gamma$, is given by

$$
\begin{aligned}
\Gamma=\frac{n_{B}}{P} \frac{d P}{d n_{B}}= & \frac{4 \mu^{4}}{3 \mu^{4}-4 \pi^{2} B_{\mathrm{eff}}}-\frac{2 B^{2} \mu^{4}}{9} \frac{\left(7-6 \log \frac{B}{2^{1 / 3} 3 \mu^{2}}\right)}{\left(3 \mu^{4}-4 \pi^{2} B_{\mathrm{eff}}\right)^{2}} \\
& -\frac{88 B^{2}}{27} \frac{B_{\mathrm{eff}} \pi^{2}}{\left(3 \mu^{4}-4 \pi^{2} B_{\mathrm{eff}}\right)^{2}}+\mathcal{O}\left(B^{4}\right),
\end{aligned}
$$

and the critical adiabatic index, $\Gamma_{c}$, for a cold star in general relativity is

$$
\Gamma_{c}=(1+w)\left[1+\frac{(3 w+1)}{2}\left[\frac{(w+1)}{6 w} \tan ^{2} \chi-1\right]\right]
$$

To get dynamical stability the condition $\Gamma>\frac{4}{3}$ must be satisfied. The intersection between $\Gamma$ and $\Gamma_{c}$ determines when a quark star becomes gravitationally unstable. We found $\Gamma_{c} \sim 2.3$ and $w_{c} \sim 0.17$ for the maximum mass value of the star (Table 2).

Another quantity that is related with the stability of the star is the speed of sound $c_{s}$. To satisfy the causality of quark matter,

$$
\frac{d P}{d \rho}=c_{s}^{2} \leqslant 1
$$

At very high densities particles become relativistic and the speed of sound should be lower, more precisely of the order of $1 / \sqrt{3}$, the speed of sound of relativistic fluids. We found

$$
c_{s}=\frac{1}{\sqrt{3}}+\frac{B^{2}}{27 \sqrt{3} \mu^{4}}+\mathcal{O}\left(B^{2}\right) .
$$

This quantity tells us how stiff is the EoS providing information about the compressibility of the fluid. The stiffness of the EoS increases when $c_{s}$ is closer to 1 . 
Table 2

Maximum Mass, Maximum Radius, and Baryonic Number for Different Bag Constants

\begin{tabular}{lcccc}
\hline \hline$B_{\text {bag }}\left(\mathrm{MeV} \mathrm{fm}^{-3}\right)$ & $B(\mathrm{G})$ & $R_{\max }(\mathrm{km})$ & $M_{\max } / M_{\odot}$ & $N_{B} / N_{\odot}$ \\
\hline 57 & 0 & 12.10 & 2.55 & 4.30 \\
& $4.4 \times 10^{17}$ & 12.06 & 2.45 & 4.09 \\
60 & 0 & 11.80 & 2.49 & 4.14 \\
& $4.8 \times 10^{17}$ & 11.76 & 2.48 & 4.14 \\
75 & 0 & 10.55 & 2.22 & 3.50 \\
& $5.3 \times 10^{17}$ & 10.52 & 2.22 & 3.50 \\
80 & 0 & 10.22 & 2.15 & 3.34 \\
& $5.5 \times 10^{17}$ & 10.18 & 2.15 & 3.34 \\
& 0 & 9.91 & 2.09 & 3.19 \\
90 & $5.7 \times 10^{17}$ & 9.89 & 2.09 & 3.19 \\
& 0 & 9.63 & 2.03 & 3.05 \\
& $5.8 \times 10^{17}$ & 9.60 & 2.03 & 3.05 \\
\hline
\end{tabular}

\section{SUMMARY AND CONCLUSIONS}

In this article, we have furnished an analytical treatment to study an HMQS in the framework of the MIT Bag model. We have analyzed the stability of quark matter with respect to strong interactions and found a restriction in the stability condition: there is a maximum value for the magnetic field beyond which quark matter becomes unstable. In the limit of "weak" magnetic field that we have studied, quark magnetic moments are aligned in the same direction of the field and this situation leads to such restriction. This could mean that if the magnetic field strength exceeds that critical value, then quark or hybrid stars should not be considered as magnetars.

In a more general case, when quark masses are taken into account, electrons should be considered. For magnetic fields much stronger than $B_{\max }$, Landau levels for electrons will increase the energy per particle. However, this probably will not contribute to modify the Iron-condition, ${ }^{56} \mathrm{Fe}=930 \mathrm{MeV}$, because electron fraction in SQM is already very low.

We have also found an analytical approximate solution for the $M-R$ relationship. Even though we used very simple physics, our results are in good agreement with the confidence contours of available observational data.

It is important to note that although the uniform energy density regime is a good approximation for quark stars, deviations in the determination of the $M-R$ relationship may occur because in the limit of high densities such approximation is no longer valid.

Finally, we calculate the adiabatic index and the speed of sound. The critical value for the adiabatic index, which corresponds to the collapse of the star, is in agreement with that of Naurenberg \& Chapline (1977), a pioneering work about quark stars. On the other hand, the speed of sound is consistent with the expected values for quark stars.

We thank A. Pérez Martínez and J. E. Horvath for comments and suggestions. M.O. acknowledges the fruitful discussion with
F. Weber and H. Rodrigues. We are grateful to A. Reisseneger for kindly and carefully answering our questions on the high energy units. We also thank S. Chakrabarty.

\section{REFERENCES}

Anand, J. D., \& Singh, S. 1999, Pramana, 52, 127

Banerjee, S., Ghosh, S. K., \& Raha, S. 2000, J. Phys. G: Nucl. Part. Phys., 26, L1

Bodmer, A. R. 1971, Phys. Rev. D, 4, 1601

Chakrabarty, S. 1996, Phys. Rev. D, 54, 1306

Chakrabarty, S., \& Sahu, P. K. 1996, Phys. Rev. D, 80, 4687

Cheng, K. S., \& Daib, Z. G. 2002, Astropart.. Phys., 16, 277

de la Incera, V. 2009, arXiv:0912.2375 [hep-ph]

Douchin, F. \& Haensel, P. 2001, A\&A, 380, 151

Drago, A., \& Lavagno, A. 2010, arXiv:1004.0325 [astro-ph]

Farhi, E., \& Jaffe, R. L. 1984, Phys. Rev. D, 30, 2379

González Felipe, R., \& Pérez Martínez, A. 2009, J. Phys. G: Nucl. Part. Phys., 36, 075202

González Felipe, R., Pérez Martínez, A., Pérez Rojas, H., \& Orsaria, M. 2008, Phys. Rev. C, 77, 015807

Harrison, B. K., Thorne, K., Wakano, M., \& Wheeler, J. A. 1965, Gravitational Theory and Gravitational Collapse (Chicago, IL: Univ. Chicago Press)

Itoh, N. 1970, Prog. Theor. Phys., 44, 291

Lattimer, J. M., \& Prakash, M. 2001, ApJ, 550, 426

Lattimer, J. M., \& Prakash, M. 2007, Phys. Rep., 442, 109

Naurenberg, M., \& Chapline, G., Jr. 1973, ApJ, 179, 277

Naurenberg, M., \& Chapline, G. 1977, Ann. New York Acad. Sci., 302, 191

Ouyed, R., Leahy, D., \& Niebergal, B. 2007, A\&A, 473, 357

Özel, F., \& Psaltis, D. 2009, Phys. Rev. D, 80, 103003

Paulucci, L., Ferrer, E. J., de la Incera, V., \& Horvath, J. E. 2011, Phys. Rev. D, 83, 043009

Pérez Martínez, A., González Felipe, R., \& Manreza Paret, D. 2010, Int. J. Mod. Phys. D, 19, 1511

Shapiro, S. L., \& Teukolsky, S. A. 1983, Black Holes, White Dwarfs, and Neutron Stars: The Physics of Compact Objects (New York: Wiley-Interscience)

Spivey, M. Z. 2006, Math. Mag., 79, 61

Weinberg, S. 1972, Gravitation and Cosmology: Principles and Applications of the General Theory of Relativity (New York: Wiley)

Witten, E. 1984, Phys. Rev. D, 30, 272

Zdunik, J. L., Bulik, T., Kluźniak, W., Haensel, P., \& Gondek-Rosińska, D. 2000, A\&A, 359, 143

Zhang, C. M., Yin, H. X., Kojima, Y., Chang, H. K., Xu, R. X., Li, X. D., Zhang, B., \& Kiziltan, B. 2007, MNRAS, 374, 232 